\title{
Depletion of ubiA prenyltransferase domain containing 1 expression promotes angiotensin II-induced hypertrophic response in AC16 human myocardial cells via modulating the expression levels of coenzyme Q10 and endothelial nitric oxide synthase
}

\author{
BINGJU YAN ${ }^{1}$, YINGXIAN SUN ${ }^{1}$ and JUN WANG ${ }^{2}$ \\ ${ }^{1}$ Department of Cardiology, The First Hospital of China Medical University, Shenyang, Liaoning 110001; \\ ${ }^{2}$ Department of Cardiology General Surgery, First Hospital of Liaoning Medical University, \\ Jinzhou, Liaoning 121000, P.R. China
}

Received August 30, 2016; Accepted May 22, 2017

DOI: $10.3892 / \mathrm{mmr} .2017 .7407$

\begin{abstract}
UbiA prenyltransferase domain containing 1 (UBIAD1) is closely associated with cardiovascular diseases. However, at the cellular level, little is known about how UBIAD1 is expressed and functions in cardiomyocyte hypertrophy. The aim of the present study was to investigate the expression and role of UBIAD1 in angiotensin II (Ang II)-induced hypertrophy in AC16 cardiomyoblast cells. The loss-of-function approach was used to knock down UBIAD1 in vehicle- and Ang II-stimulated AC16 cells. The levels of atrial natriuretic factor (ANF) and caspase-3 were measured and compared between vehicle- and Ang II-treated AC16 cells pretreated with control siRNA or siRNA against UBIAD1. In addition, the levels of coenzyme Q10 (CoQ10) and endothelial nitric oxide synthase (eNOS) were evaluated and compared between these groups. Ang II induced hypertrophy and apoptosis in AC16 cells, accompanied by increased expression of ANF and caspase-3, and decreased expression of UBIAD1. These effects were potentiated by UBIAD1 knockdown. In addition, Ang II treatment suppressed the expression of CoQ10 and eNOS, as well as the production of NO, and these inhibitory effects were also enhanced by UBIAD1 knockdown. Thus, silencing of UBIAD1 expression promotes a myocardial hypertrophic response to Ang II stimulation, in part, by suppressing the expression of CoQ10 and eNOS.
\end{abstract}

Correspondence to: Dr Yingxian Sun, Department of Cardiology, The First Hospital of China Medical University, 155 Nanjing North Street, Heping, Shenyang, Liaoning 110001, P.R. China

E-mail: yxsun@cmu.edu.cn

Key words: ubiA prenyltransferase domain containing 1, angiotensin II, coenzyme Q10, endothelial nitric oxide synthase

\section{Introduction}

Cardiac hypertrophy is a cardiac muscle disorder that may eventually lead to heart failure, regardless of its etiology. Cardiac hypertrophy is a risk factor for cardiovascular mortality and morbidity independently or through interacting with other cardiovascular risk factors. Hence, controlling hypertrophic remodeling may offer the most promising novel therapeutic strategy for reducing cardiovascular morbidity and mortality in cardiac hypertrophy and heart failure. The vast majority of existing therapeutic strategies exert antihypertrophic effects via targeting extracellular receptors in cardiac cells, but their efficacy appears to be limited. Attention has turned to targeting intracellular signaling pathways in the last decade (1).

Coenzyme Q10 or ubiquinone-10 (CoQ10), the only endogenously synthesized lipid-soluble antioxidant, has been demonstrated to be important in maintaining normal cardiovascular function in humans and a mouse disease model (2-4). For example, a decreased expression level of CoQ10 was observed in human cardiomyopathy (5) and CoQ10 significantly ameliorated cardiac function in children with dilated cardiomyopathy (6). In a mouse diabetic model, CoQ10 has been shown to efficiently diminish cardiac hypertrophy and fibrosis (7). Furthermore, CoQ10 has been widely used as a daily supplement to benefit cardiovascular function (5). In addition, CoQ10 is involved in $\mathrm{NAD}(\mathrm{P}) \mathrm{H}$-oxidoreductase-dependent reactions, such as the synthesis of nitric oxide (NO) (8), which is generated by endothelial NO synthase (eNOS), a well-known mediator of cardiovascular homeostasis $(9,10)$. As in the case with non-mitochondrial CoQ10, which is predominantly present in the Golgi and plasma membrane $(11,12)$, eNOS is also specifically localized in the Golgi and plasma membrane of heart and endothelial cells, and its function is differentially regulated in these two cellular compartments (13). Previous studies have shown that CoQ10 improves endothelial dysfunction, in part, by 'recoupling' eNOS and modulating NO-associated signaling (14).

UbiA prenyltransferase domain containing 1 (UBIAD1; also termed transitional epithelial response protein 1), belongs 
to the UbiA superfamily of prenyltransferases (15). UBIAD1 was first identified as an enzyme that catalyzes the synthesis of vitamin K2 $(16,17)$, a well-known factor involved in the maintenance of cardiovascular homeostasis. Previously, UBIAD1 has been reported to synthesize CoQ10 and exhibit anti-oxidant activity (18). Although UBIAD1 was first shown to be potentially involved in the development of bladder and prostate tumors (19), emerging evidence indicates that UBIAD1 is essential in normal cardiovascular development and function. For example, a UBIAD1 mutation has been associated with cardiac edema and vascular phenotypes in zebrafish, which were rescued by the re-expression of human UBIAD1 (20). In a mouse model, UBIAD1 knockout led to embryonic death at E7.5-E10.5; however, whether there were any cardiovascular phenotypes was not reported (21). Given that UBIAD1 contributes significantly to the generation of non-mitochondrial CoQ (18), it is presumed that UBIAD1 contributes to mitochondrial heart disease. However, whether and how UBIAD1 is involved in mediating cardiomyocyte hypertrophy in response to agonists remains unknown.

Given the above findings that link UBIAD1 to CoQ10, it was hypothesized in the present study that UBIAD1 may be involved in mediating stressor-induced cardiac hypertrophy via mediating CoQ10 expression levels. The current study aimed to determine the role and potential underlying mechanism of UBIAD1 in angiotensin II (Ang II)-induced myocardial hypertrophy in vitro.

\section{Materials and methods}

Cell culture and treatments. AC16 human myocardial cells (Guangzhou Lede Kangkang Biotechnology Co., Ltd., Guangzhou, Guangdong, China) were cultured in Dulbecco's modified Eagle's medium supplemented with $10 \%$ fetal bovine serum (HyClone; GE Healthcare Life Sciences, Chicago, IL, USA), $2 \mathrm{mM}$ glutamine, $100 \mathrm{U} / \mathrm{ml}$ penicillin, $100 \mu \mathrm{g} / \mathrm{ml}$ streptomycin, and $1 \mathrm{mM}$ pyruvate in humidified air $\left(5 \% \mathrm{CO}_{2}\right)$ at $37^{\circ} \mathrm{C}$. The $\mathrm{AC} 16$ cells were transiently transfected with UBIAD1 siRNA (Si-UBIAD1; Si-UBIAD1 was tagged with a carboxyfluorescein green fluorophore) or a negative control siRNA (NC; Shanghai GenePharma Co.,Ltd., Shanghai,China) using Invitrogen Lipofectamine 2000 reagent (Thermo Fisher Scientific, Inc., Waltham, MA, USA), according to the manufacturer's protocol. The sequences of the UBIAD1-siRNA were as follows: Forward, 5'-CACUUGGCUCUUAUCUACUdT dT-3' and reverse, 5'-AGUAGAUAAGAGCCAAGUGdTdT-3'. The sequences of the NC were as follows: Forward, 5'-UUC UCCGAACGUGUCACGUTT-3' and reverse, 5'-ACGUGA CACGUUCGGAGAATT-3'.

Measurement of the surface area. Motic Images 1.3 software (Beijing Hanmengzixing Instruments and Meters Co., Ltd., Beijing, China) was used to measure the surface area of the AC16 cells. Five fields were randomly selected for each treatment, and $15-25$ cells/field were measured.

3-(4,5-Dimethylthiazol-2-yl)-2,5-diphenyltetrazolium bromide (MTT) assay. The MTT assay was performed to analyze the viability of the AC16 cells. Briefly, AC16 cells were plated at a density of $1 \times 10^{5} / \mathrm{ml}$ in a 96 -well plate for $24 \mathrm{~h}$, transfected with Si-UBIAD1 or NC, followed by treatment with vehicle or Ang II for $24 \mathrm{~h}$ (Sigma-Aldrich; Merck KGaA, Darmstadt, Germany). The AC16 cells were incubated with $20 \mu \mathrm{lof} 5 \mathrm{mg} / \mathrm{ml}$ MTT solution (HyClone; GE Healthcare Life Sciences) for $4 \mathrm{~h}$ at $37^{\circ} \mathrm{C}$. Thereafter, $150 \mu \mathrm{l}$ dimethyl sulfoxide (HyClone; GE Healthcare Life Sciences) was added to each well to dissolve the crystal formazan dye, and the plate was agitated for $10 \mathrm{~min}$ until all the crystals were dissolved. The quantity of MTT formazan was determined by the absorbance at a wavelength of $490 \mathrm{~nm}$ using a microplate reader.

Determination of apoptosis with flow cytometry. The rate of cell apoptosis was analyzed with flow cytometry (BD FACSCanto $^{\mathrm{TM}}$ II system; BD Biosciences, Franklin Lakes, NJ, USA) following FITC-Annexin V and propidium iodide (PI) staining. The staining procedure was performed according to the instructions of the Annexin V-FITC Apoptosis Detection kit (BioVision, Inc., Milpitas, CA, USA). Briefly, AC16 cells were resuspended following centrifugation to a concentration of $1 \times 10^{6}$ cells $/ \mathrm{ml}$. A total of $100 \mu 1$ cells were transferred to $5 \mathrm{ml}$ tubes, followed by addition of $5 \mu \mathrm{l}$ FITC-Annexin $\mathrm{V}$ and $5 \mu \mathrm{l}$ PI. The cell suspension was gently vortexed and incubated at room temperature for $15 \mathrm{~min}$, shielded from light, and then $400 \mu \mathrm{l}$ Annexin V binding buffer was added. Flow cytometry was performed within $1 \mathrm{~h}$. The following method was applied: The Annexin V-negative/PI-negative fraction represented viable cells; the Annexin V-positive/PI-negative fraction represented early apoptotic cells; and the Annexin V-positive/PI-positive fraction represented late apoptotic and dead cells.

Reverse transcription-quantitative polymerase chain reaction $(R T-q P C R)$. Total RNA was isolated using the PureLink Micro-to-Midi Total RNA Purification system (Invitrogen; Thermo Fisher Scientific, Inc.) and cDNA was synthesized with an RT High Capacity kit (Applied Biosystems; Thermo Fisher Scientific, Inc.) according to the manufacturer's protocol. RT-qPCR was performed with an ABI Prism 7300 Real-Time PCR system (Applied Biosystems; Thermo Fisher Scientific, Inc.) using Platinum Quantitative PCR SuperMix-UDG with ROX (Invitrogen; Thermo Fisher Scientific, Inc.). The primers used in the RT-qPCR were as follows: Forward, 5'-AACGACTGTCCCGAGCAA-3' and reverse, 5'-CGGCACAACCCACCAA-3' for UBIAD1; forward, 5'-AGCCGAGACAGCAAACA-3' and reverse, 5'-GCCTGG GAGCCAAAA-3' for atrial natriuretic factor (ANF); forward, 5'-AGAGCTGGACTGCGGTATTGAG-3' and reverse, 5'-GAACCATGACCCGTCCCTTG-3' for caspase-3; forward, 5'-GACCATAATGCCTCACC-3' and reverse, 5'-ATGCGT TCATCACCAA-3' for CoQ10; forward, 5'-GCAGAGGAG TCCAGCGAACA-3' and reverse, 5'-TGGGTGCTGAGCTGA CAGAGTA-3' for eNOS; and forward, 5'-GAGGCTCTCTTC CAGCCTTC-3' and reverse, 5'-AGGGTGTAAAAGCAG CTCA-3' for GAPDH. The thermocycling conditions were as follows: $50^{\circ} \mathrm{C}$ for $2 \mathrm{~min}$, then $10 \mathrm{~min}$ at $95^{\circ} \mathrm{C}$, followed by 40 cycles of $95^{\circ} \mathrm{C}$ for $30 \mathrm{sec}$ and $55^{\circ} \mathrm{C}$ for $30 \mathrm{sec}$. The relative expression was assessed using the $2^{-\Delta \mathrm{Cq}}$ method, and converted to fold changes using the $2^{-\Delta \Delta \mathrm{Cq}}$ method (22).

Western blot analysis. Whole cell lysates were extracted from AC16 cells using RIPA lysis buffer, according to the 
A

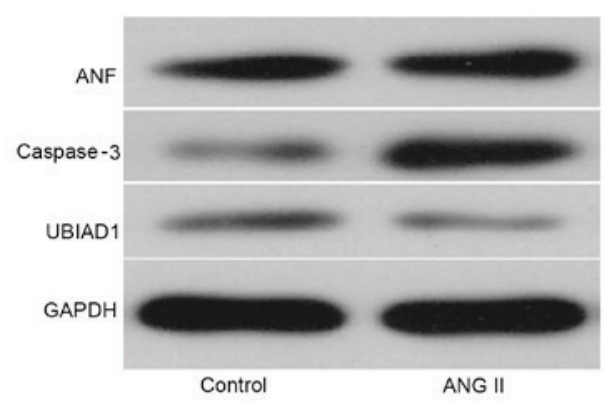

c

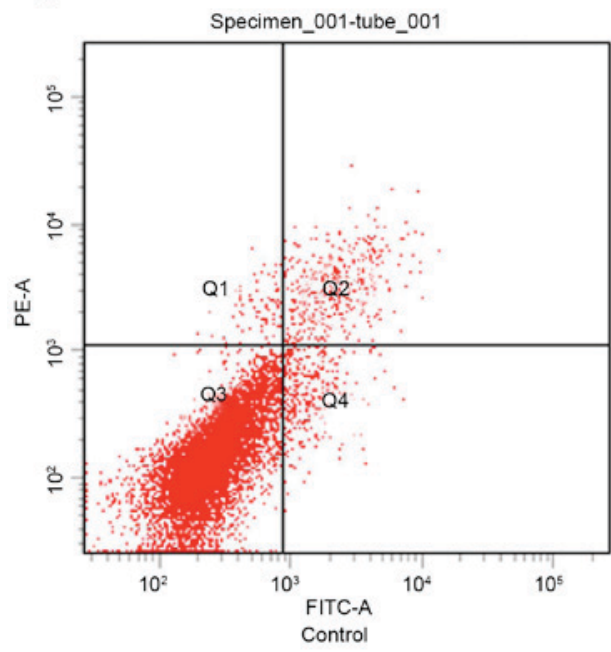

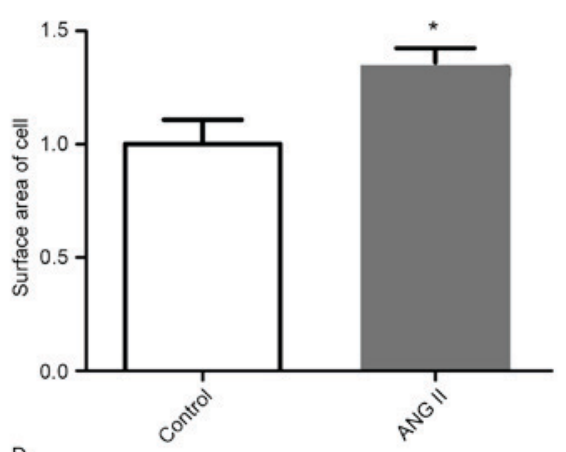

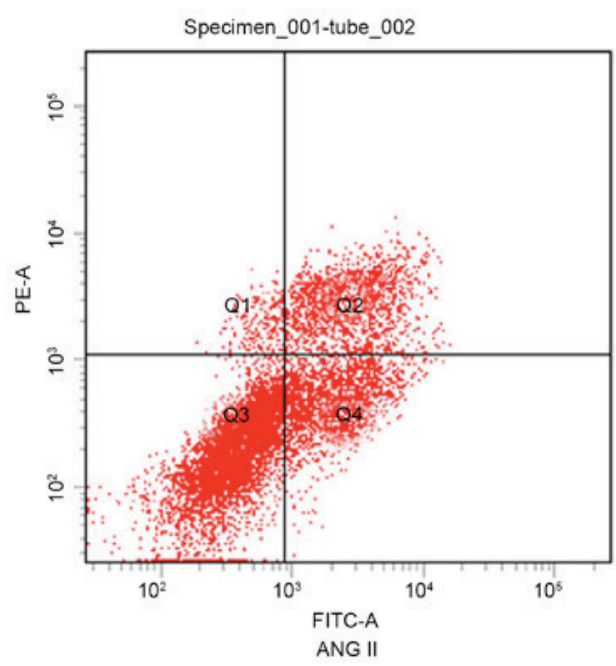

Figure 1. Ang II induced hypertrophy and apoptosis in AC16 cells. (A) Ang II increased the expression levels of ANF and caspase-3, but decreased UBIAD1 expression levels. Western blot was performed in cell lysates purified from vehicle-(control) and Ang II-stimulated AC16 cells. GAPDH served as a loading control. (B) Ang II induced hypertrophy of AC16 cells and the surface area was measured. Data are presented as the mean \pm standard error mean. ${ }^{*} \mathrm{P}<0.05$ vs. control. (C and D) Ang II induced apoptosis in AC16 cells as demonstrated using flow cytometry. Quadrants 2 and 4 represent cells undergoing apoptosis. Quadrant 1 represents normal cells and Quadrant 3 represents dead cells. Ang II, angiotensin II; ANF, atrial natriuretic factor; UBIAD1, ubiA prenyltransferase domain containing 1.

manufacturer's instructions (Abcam, Cambridge, UK) and quantified using a Bicinchoninic Acid Protein Assay kit (Bio-Rad Laboratories, Inc., Hercules, CA, USA). A total of $20 \mu \mathrm{g}$ protein samples were separated by $10 \%$ sodium dodecyl sulfate-polyacrylamide gel electrophoresis and transferred (70 V, $90 \mathrm{~min}$ ) onto a polyvinylidene difluoride membrane (EMD Millipore, Billerica, MA, USA). The membrane was blocked in Tris-buffered saline containing 5\% nonfat milk and $0.1 \%$ Tween-20 for $1 \mathrm{~h}$ at room temperature, followed by an incubation with the primary antibody against GAPDH $(1: 5,000$; cat no. 5174; Cell Signaling Technology, Inc., Danvers, MA, USA), UBIAD1 (1:1,000; cat no. ab191691; Abcam), ANF (1:500; cat no. sc-80686), and caspase-3 (1:1,000; cat no. sc-7272) (both from Santa Cruz Biotechnology, Inc., Dallas, TX, USA), CoQ10 (1:1,000; cat no. 17812-1-AP) and eNOS (1:1,000; cat no. 20116-1-AP) (both from Wuhan Sanying Biotechnology, Wuhan, China) at $4^{\circ} \mathrm{C}$ overnight. Following washing with TBST (0.1\% Tween-20), the membrane was incubated with a peroxidase-conjugated secondary antibody (1:400; cat no. A0216; Beyotime Institute of Biotechnology, Haimen, China) for $1 \mathrm{~h}$ at room temperature. Protein bands were visualized using the ECL detection system (GE Healthcare Life Sciences). Image analysis and quantification were performed using a scanning densitometer.
Measurement of NO production. The accumulation of NO was assayed using the Griess reagent. Briefly, the cells were transfected with Si-UBIAD1 or NC, and then treated with Ang II for $24 \mathrm{~h}$. Thereafter, $100 \mu \mathrm{l}$ Griess reagent was combined with an equal volume of cell supernatant and incubated at room temperature for $5 \mathrm{~min}$. The optical density at $540 \mathrm{~nm}$ was measured, and the concentration of nitrite was calculated according to the standard curve generated from known concentrations of sodium nitrite (14).

Statistical analysis. Data were expressed as the mean \pm standard error of the mean. Statistical differences were assessed by one way-analysis of variance, followed by the Duncan test for comparison between groups. $\mathrm{P}<0.05$ was considered to indicate a statistically significant difference.

\section{Results}

Ang II increased the expression of ANF and caspase-3, and decreased UBIAD1 expression in AC16 cells. Western blot analysis was performed in cell lysates purified from AC16 cells stimulated with either vehicle or ANG II (100 nM) for $24 \mathrm{~h}$. As shown in Fig. 1A, Ang II markedly increased ANF and caspase-3 expression, compared with the vehicle 

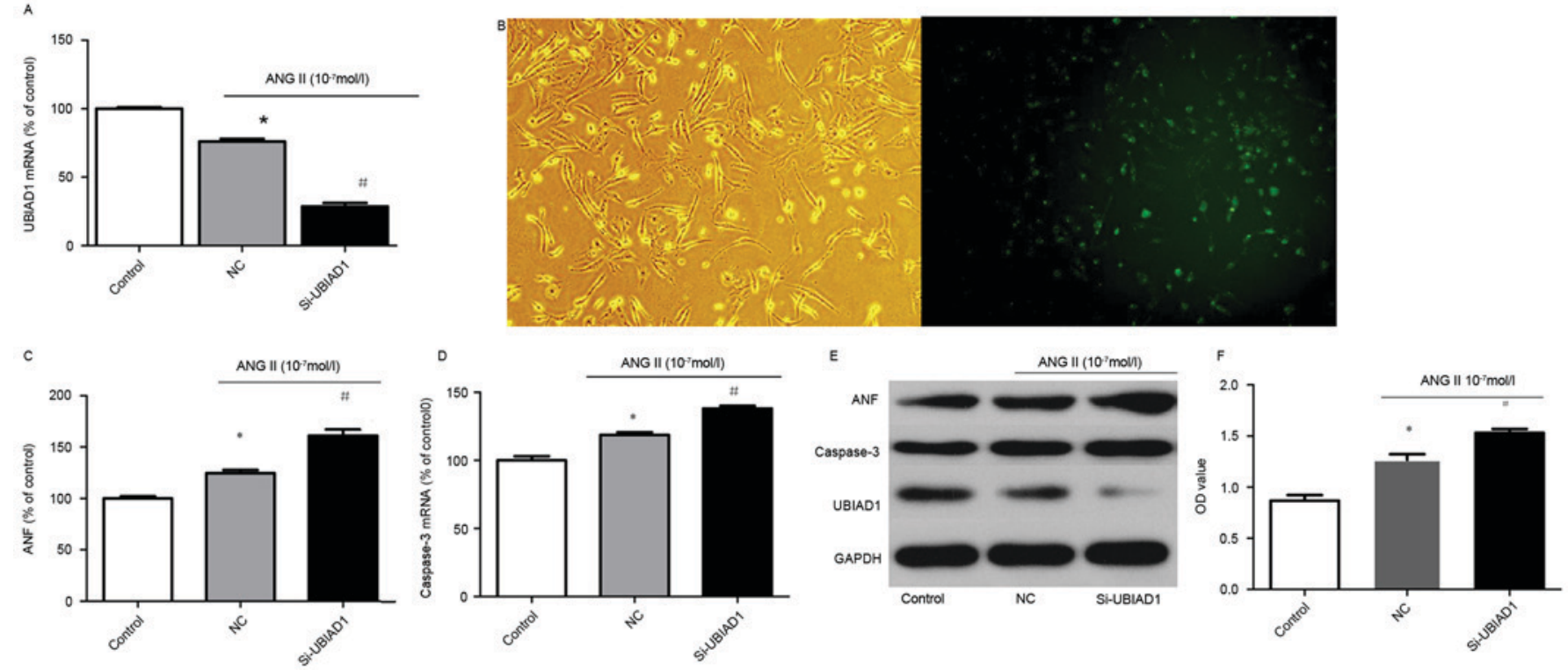

Figure 2. Knockdown of UBIAD1 potentiated the Ang II-induced increase of ANF and caspase-3 in AC16 cells. (A) Si-UBIAD1 significantly suppressed the expression of UBIAD1.(B) Si-UBIAD1 was successfully transfected with an efficacy of 95\% (tagged with carboxyfluorescein green fluorophore; magnification, x100). Depletion of UBIAD1 further attenuated the Ang II-induced decrease in UBIAD1 expression. Depletion of UBIAD1 potentiated the Ang II-induced increase of (C) ANF and (D) caspase-3 in AC16 cells. (E) Western blot analysis demonstrated that UBIAD1 knockdown potentiated the protein expression levels of ANF and caspase-3, but further decreased the UBIAD1 expression levels in Ang II-treated AC16 cells. (F) UBIAD1 knockdown increased cell viability. Data are presented as the mean \pm standard error mean. ${ }^{*} \mathrm{P}<0.05$ vs. control; ${ }^{*} \mathrm{P}<0.05$ vs. NC. UBIAD1, ubiA prenyltransferase domain containing 1 ; Ang II, angiotensin II; ANF, atrial natriuretic factor; NC, negative control.

group. In addition, the level of UBIAD1 in the AC16 cells of these two stimulation groups was measured and UBIAD1 was observed to be markedly decreased in Ang II-stimulated cells, compared with that in vehicle-treated cells. The surface area of the AC16 cells stimulated by Ang II or vehicle was measured using Motic Images 1.3. As shown in Fig. 1B, Ang II stimulation significantly increased the cell size by 1.46 times, compared with the vehicle treatment group $(\mathrm{P}<0.05)$. As caspase-3 expression was elevated by Ang II stimulation, whether apoptosis also increased in Ang II-stimulated AC16 cells was subsequently investigated. Indeed, flow cytometry experiments revealed that more apoptotic cells were observed in Ang II-treated AC16 cells than in vehicle-treated cells (Fig. 1C and D).

Depletion of UBIADI increased ANG II-stimulated expression of ANF and caspase-3 in AC16 cells. Next, whether UBIAD1 was involved in the Ang II-induced elevated expression of ANP and caspase-3 in AC16 cells was investigated. The expression level of UBIAD1 in vehicle- and Ang II-treated AC16 cells was quantitatively assessed by RT-qPCR. Ang II stimulation significantly decreased UBIAD1 mRNA expression (Fig. 2A).

Subsequently, to investigate whether UBIAD1 contributed to the hypertrophic response of AC16 cells to Ang II stimulation, knock down of UBIAD1 in AC16 cells was performed with Si-UBIAD1. Compared with AC16 cells transfected with control siRNA (the NC), Si-UBIAD1 markedly diminished the expression of UBIAD1 with an efficacy of $\sim 95 \%$ (Fig. 2B). Furthermore, downregulation of UBIAD1 further promoted the increased expression of ANF and caspase- 3 induced by Ang II at the mRNA and protein expression levels (Fig. 2C-E). In addition, Si-UBIAD1 depletion significantly increased the number of viable cells in the Ang II-stimulated group as measured by the MTT assay (Fig. 2F).

UBIADI knockdown potentiated the Ang II-induced decrease in the expression of CoQ10 and eNOS. To understand the mechanisms by which knockdown of UBIAD1 potentiated the molecular phenotypes induced by Ang II stimulation in AC16 cells, the protein and mRNA expression levels of CoQ10 and eNOS, which are key regulators of cardiovascular homeostasis were analyzed. Fig. 3A and B indicate that knockdown of UBIAD1 further attenuated the decrease in mRNA expression of CoQ10 and eNOS in Ang II-stimulated AC16 cells. Consistent with this finding, the protein expression levels of CoQ10 and eNOS were also further decreased in Ang II-treated cells transfected with NC (Fig. 3C).

UBIADI knockdown potentiated the Ang II-induced decrease in NO production. The levels of NO in vehicle- and Ang II-treated AC16 cells that were transfected with $\mathrm{NC}$ and Si-UBIAD1, respectively were also measured. As shown in Fig. 4, the NO level was decreased in Ang II-stimulated AC16 cells, compared with the vehicle-treated cells $(\mathrm{P}<0.05)$. UBIAD1 knockdown further attenuated this decrease $(\mathrm{P}<0.05)$.

\section{Discussion}

In the present study, the potential involvement of UBIAD1 in Ang II-induced hypertrophy was investigated in AC16 cells, a human ventricular cardiac cell line. It was demonstrated that Ang II stimulation increased the expression of ANF and caspase-3, which coincided with the decreased expression of UBIAD1. Knockdown of UBIAD1 further potentiated the Ang II-triggered decrease in the levels of ANF 
A

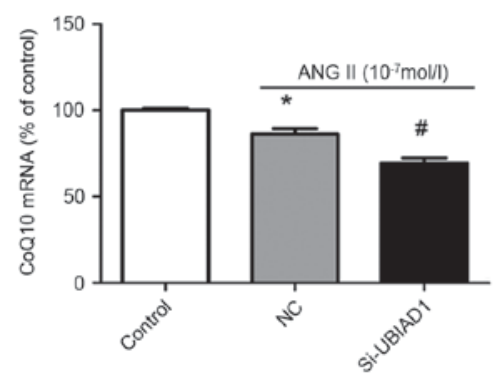

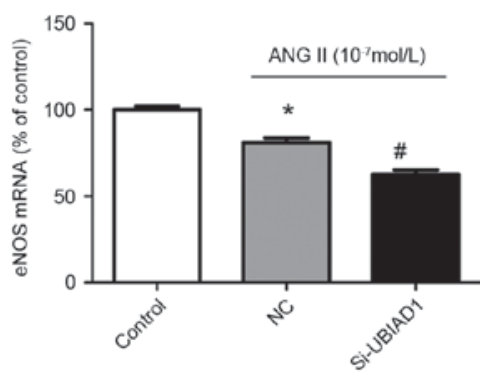

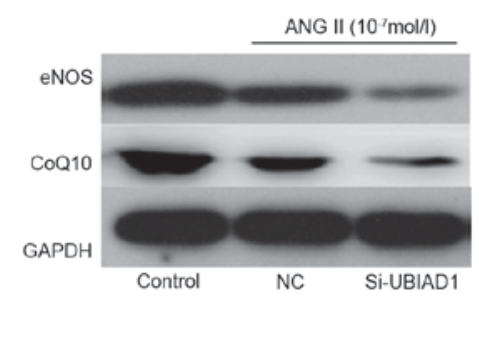

Figure 3. Knockdown of UBIAD1 further suppressed the decreased expression levels of CoQ10 and eNOS by Ang II stimulation. (A and B) Reverse transcription-quantitative polymerase chain reaction was performed to evaluate the mRNA expression levels of CoQ10 and eNOS in the control (vehicle-treated) group, NC (i.e. the control siRNA-treated group) and Si-UBIAD1 cells. (C) Western blotting demonstrated a further decrease in the protein levels of CoQ10 and eNOS in Ang II-stimulated Si-UBIAD1 cells, compared with NC cells. Data are presented as the mean \pm standard error mean. ${ }^{*} \mathrm{P}<0.05$ vs. control; ${ }^{\#} \mathrm{P}<0.05$ vs. NC. UBIAD1, ubiA prenyltransferase domain containing 1; CoQ10, coenzyme Q10; eNOS, endothelial nitric oxide synthase; Ang II, angiotensin II; ANF, atrial natriuretic factor; $\mathrm{NC}$, negative control.

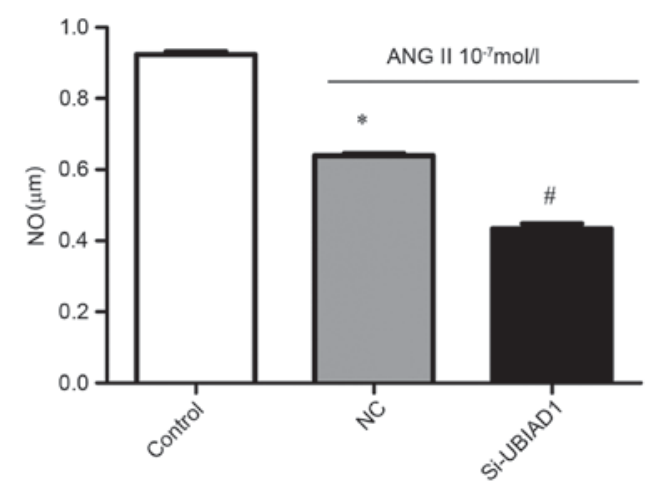

Figure 4. Knockdown of UBIAD1 further suppressed NO production in Ang II-stimulated AC16 cells. Data are presented as the mean \pm standard error mean. ${ }^{*} \mathrm{P}<0.05$ vs. control (vehicle-treated group); ${ }^{\#} \mathrm{P}<0.05$ vs. $\mathrm{NC}$ (the control siRNA-treated group). UBIAD1, ubiA prenyltransferase domain containing 1; NO, nitric oxide; Ang II, angiotensin II; NC, negative control.

and caspase-3. Mechanistically, downregulation of UBIAD1 in Ang II-stimulated AC16 cells was associated with a further decrease in the expression of CoQ10 and eNOS, as well as the production of NO induced by Ang II.

Ang II is important in the pathophysiology of cardiovascular diseases and metabolic disorders (23-25). Evidence from clinical trials, animal models, and cellular studies indicates that renin-angiotensin system inhibitors, including renin inhibitors, Ang II type 1 receptor blockers, and Ang-converting enzyme inhibitors, improve cardiac function and ameliorate cardiac hypertrophy (26-29). eNOS and its product, NO are also involved in Ang II-induced pathophysiological changes in the cardiovascular system (30). In addition, accumulating evidence indicates that reduced NO bioavailability contributes to the pathogenesis of cardiac hypertrophy and heart failure (31). In our model system, Ang II stimulation inhibited the expression of eNOS and NO production, which coincided with the decrease in UBIAD1 expression. Additionally, depletion of UBIAD1 further enhanced the Ang II-triggered inhibition of eNOS and NO, indicating that UBIAD1 potentially antagonizes the repressive impact of Ang II on eNOS and NO expression/production. In addition, a significant decrease in CoQ10 expression was observed in Ang II-treated cells, compared with the vehicle-treated cells. Given that CoQ10 offers a variety of benefits to the cardiovascular system against oxidative stress, a decrease in CoQ10 level is detrimental to cellular function. However, CoQ10 has been shown to ameliorate the clinical manifestations of patients with hypertrophic cardiomyopathy (32). Furthermore, depletion of UBIAD1 further attenuated the decrease in CoQ10 by Ang II, which is consistent with the recent finding that UBIAD1 is involved in the synthesis of CoQ10 (18).

Caspase-3 and ANF are representative indicators for cardiac hypertrophy and apoptosis, respectively. In the current study, Ang II (100 nM) stimulation for $24 \mathrm{~h}$ induced hypertrophy and apoptosis in AC16 cells, which was consistent with studies on animal models and other cell lines $(30,33)$. Silencing of UBIAD1 by siRNA further potentiated the increase in expression levels of ANF and caspase-3 elicited by Ang II treatment, indicating that UBIAD1 is negatively implicated in the Ang II-induced expression of ANF and caspase- 3 in AC16 cells. Furthermore, the MTT assay for cell viability demonstrated that the optical density of silenced UBIAD1 myocardial cells was higher than that of the negative control group.

Missense mutations of the UBIAD1 gene are etiologically linked to the genetic disorder Schnyder corneal dystrophy (SCD), in which the accumulation of cholesterol and phospholipids occurs in the cornea of the eye, eventually leading to blindness (34). However, no cardiac phenotypes in patients with SCD have been reported. Given that UBIAD1 is implicated in the generation of CoQ10 and eNOS, which are important for cardiovascular homeostasis, it will be interesting to clarify whether a mutation of the UBIAD1 gene predisposes humans or mice to cardiac muscle disorders in response to external stressors.

In conclusion, the present study demonstrated that Ang II stimulation induces hypertrophy in AC16 cardiac cells, accompanied by increased apoptosis and expression levels of the cardiac disease marker ANF. In addition, Ang II treatment suppresses the expression levels of eNOS and CoQ10, which have beneficial effects on cardiomyocyte functions. Silencing UBIAD1 further aggravates the deleterious effects rendered by Ang II, indicating that a normal or increased level of UBIAD1 may offer protection against the Ang II-induced hypertrophic response and apoptosis. Thus, the current 
findings provide novel insight into the potential protection of UBIAD1 in cardiac hypertrophy and its underlying molecular mechanisms.

\section{References}

1. McKinsey TA and Kass DA: Small-molecule therapies for cardiac hypertrophy: Moving beneath the cell surface. Nat Rev Drug Discov 6: 617-635, 2007.

2. Kumar A, Kaur H, Devi P and Mohan V: Role of coenzyme Q10 (CoQ10) in cardiac disease, hypertension and Meniere-like syndrome. Pharmacol Ther 124: 259-268, 2009.

3. Crane FL: Discovery of ubiquinone (coenzyme Q) and an overview of function. Mitochondrion 7 (Suppl): S2-S7, 2007.

4. Bentinger M, Tekle M and Dallner G: Coenzyme Q-biosynthesis and functions. Biochem Biophys Res Commun 396: 74-79, 2010.

5. Saha SP and Whayne TF Jr: Coenzyme Q-10 in human health: Supporting evidence? South Med J 109: 17-21, 2016.

6. Kocharian A, Shabanian R, Rafiei-Khorgami M, Kiani A and Heidari-Bateni G: Coenzyme Q10 improves diastolic function in children with idiopathic dilated cardiomyopathy. Cardiol Young 19: 501-506, 2009.

7. Huynh K, Kiriazis H, Du XJ, Love JE, Jandeleit-Dahm KA, Forbes JM, McMullen JR and Ritchie RH: Coenzyme Q10 attenuates diastolic dysfunction, cardiomyocyte hypertrophy and cardiac fibrosis in the $\mathrm{db} / \mathrm{db}$ mouse model of type 2 diabetes. Diabetologia 55: 1544-1553, 2012.

8. Navas P, Villalba JM and de Cabo R: The importance of plasma membrane coenzyme $\mathrm{Q}$ in aging and stress responses. Mitochondrion 7 (Suppl): S34-S40, 2007.

9. Alp NJ and Channon KM: Regulation of endothelial nitric oxide synthase by tetrahydrobiopterin in vascular disease. Arterioscler Thromb Vasc Biol 24: 413-420, 2004.

10. Forsgren M, Attersand A, Lake S, Grünler J, Swiezewska E, Dallner $\mathrm{G}$ and Climent I: Isolation and functional expression of human COQ2, a gene encoding a polyprenyl transferase involved in the synthesis of CoQ. Biochem J 382: 519-526, 2004.

11. Bentinger M, Tekle M, Brismar K, Chojnacki T, Swiezewska E and Dallner G: Stimulation of coenzyme Q synthesis. Biofactors 32: 99-111, 2008.

12. Kalén A, Appelkvist EL, Chojnacki T and Dallner G: Nonaprenyl-4-hydroxybenzoate transferase, an enzyme involved in ubiquinone biosynthesis, in the endoplasmic reticulum-Golgi system of rat liver. J Biol Chem 265: 1158-1164, 1990.

13. Fulton D, Fontana J, Sowa G, Gratton JP, Lin M, Li KX, Michell B, Kemp BE, Rodman D and Sessa WC: Localization of endothelial nitric-oxide synthase phosphorylated on serine 1179 and nitric oxide in Golgi and plasma membrane defines the existence of two pools of active enzyme. J Biol Chem 277: 4277-4284, 2002.

14. Tsai KL, Huang YH, Kao CL, Yang DM, Lee HC, Chou HY, Chen YC, Chiou GY, Chen LH, Yang YP, et al: A novel mechanism of coenzyme Q10 protects against human endothelial cells from oxidative stress-induced injury by modulating NO-related pathways. J Nutr Biochem 23: 458-468, 2012.

15. McGarvey TW, Nguyen T, Puthiyaveettil R, Tomaszewski JE and Malkowicz SB: TERE1, a novel gene affecting growth regulation in prostate carcinoma. Prostate 54: 144-155, 2003.

16. Nakagawa K, Hirota Y, Sawada N, Yuge N, Watanabe M, Uchino Y, Okuda N, Shimomura Y, Suhara Y and Okano T: Identification of UBIAD1 as a novel human menaquinone-4 biosynthetic enzyme. Nature 468: 117-121, 2010.

17. Hirota Y, Tsugawa N, Nakagawa K, Suhara Y, Tanaka K, Uchino Y, Takeuchi A, Sawada N, Kamao M, Wada A, et al: Menadione (vitamin K3) is a catabolic product of oral phylloquinone (vitamin K1) in the intestine and a circulating precursor of tissue menaquinone-4 (vitamin K2) in rats. J Biol Chem 288: 33071-33080, 2013.

18. Mugoni V, Postel R, Catanzaro V, De Luca E, Turco E, Digilio G Silengo L, Murphy MP, Medana C, Stainier DY, et al: Ubiad1 is an antioxidant enzyme that regulates eNOS activity by CoQ10 synthesis. Cell 152: 504-518, 2013

19. McGarvey TW, Nguyen T, Tomaszewski JE, Monson FC and Malkowicz SB: Isolation and characterization of the TERE1 gene, a gene down-regulated in transitional cell carcinoma of the bladder. Oncogene 20: 1042-1051, 2001.
20. Hegarty JM, Yang H and Chi NC: UBIAD1-mediated vitamin $\mathrm{K} 2$ synthesis is required for vascular endothelial cell survival and development. Development 140: 1713-1719, 2013.

21. Nakagawa K, Sawada N, Hirota Y, Uchino Y, Suhara Y, Hasegawa T, Amizuka N, Okamoto T, Tsugawa N, Kamao M, et al: Vitamin K2 biosynthetic enzyme, UBIAD1 is essential for embryonic development of mice. PLoS One 9: e104078, 2014.

22. Livak KJ and Schmittgen TD: Analysis of relative gene expression data using real-time quantitative PCR and the 2(-Delta Delta C(T)) method. Methods 25: 402-408, 2001.

23. Cooper SA, Whaley-Connell A, Habibi J, Wei Y, Lastra G, Manrique C,Stas S and Sowers JR: Renin-angiotensin-aldosterone system and oxidative stress in cardiovascular insulin resistance. Am J Physiol Heart Circ Physiol 293: H2009-H2023, 2007.

24. Nakashima H, Suzuki H, Ohtsu H, Chao JY, Utsunomiya H, Frank GD and Eguchi S: Angiotensin II regulates vascular and endothelial dysfunction: Recent topics of Angiotensin II type-1 receptor signaling in the vasculature. Curr Vasc Pharmacol 4: 67-78, 2006.

25. Olivares-Reyes JA, Arellano-Plancarte A and Castillo-Hernandez JR: Angiotensin II and the development of insulin resistance: Implications for diabetes. Mol Cell Endocrinol 302: 128-139, 2009.

26. Carvalheira JB, Calegari VC, Zecchin HG, Nadruz W Jr, Guimarães RB, Ribeiro EB, Franchini KG, Velloso LA and Saad MJ: The cross-talk between angiotensin and insulin differentially affects phosphatidylinositol 3-kinase- and mitogen-activated protein kinase-mediated signaling in rat heart: Implications for insulin resistance. Endocrinology 144: 5604-5614, 2003.

27. Habibi J, Whaley-Connell A, Hayden MR, DeMarco VG, Schneider R, Sowers SD, Karuparthi P, Ferrario CM and Sowers JR: Renin inhibition attenuates insulin resistance, oxidative stress, and pancreatic remodeling in the transgenic Ren2 rat. Endocrinology 149: 5643-5653, 2008.

28. Koh KK, Quon MJ, Lee Y, Han SH, Ahn JY, Chung WJ, Kim JA and Shin EK: Additive beneficial cardiovascular and metabolic effects of combination therapy with ramipril and candesartan in hypertensive patients. Eur Heart J 28: 1440-1447, 2007.

29. Lastra G, Whaley-Connell A, Manrique C, Habibi J, Gutweiler AA, Appesh L, Hayden MR, Wei Y, Ferrario C and Sowers JR: Low-dose spironolactone reduces reactive oxygen species generation and improves insulin-stimulated glucose transport in skeletal muscle in the TG(mRen2)27 rat. Am J Physiol Endocrinol Metab 295: E110-E116, 2008.

30. Lin LY, Lin CY, Su TC and Liau CS: Angiotensin II-induced apoptosis in human endothelial cells is inhibited by adiponectin through restoration of the association between endothelial nitric oxide synthase and heat shock protein 90 . FEBS Lett 574: 106-110, 2004

31. Landmesser U, Engberding N, Bahlmann FH, Schaefer A Wiencke A, Heineke A, Spiekermann S, Hilfiker-Kleiner D, Templin C, Kotlarz D, et al: Statin-induced improvement of endothelial progenitor cell mobilization, myocardial neovascularization, left ventricular function, and survival after experimental myocardial infarction requires endothelial nitric oxide synthase. Circulation 110: 1933-1939, 2004.

32. Langsjoen PH, Langsjoen A, Willis R and Folkers K: Treatment of hypertrophic cardiomyopathy with coenzyme Q10. Mol Aspects Med 18 (Suppl): S145-S151, 1997.

33. Lakó-Futó Z, Szokodi I, Sármán B, Földes G, Tokola H, Ilves M, Leskinen H, Vuolteenaho O, Skoumal R, deChâtel R, et al: Evidence for a functional role of angiotensin II type 2 receptor in the cardiac hypertrophic process in vivo in the rat heart. Circulation 108: 2414-2422, 2003.

34. Orr A, Dubé MP, Marcadier J, Jiang H, Federico A, George S, Seamone C, Andrews D, Dubord P, Holland S, et al: Mutations in the UBIAD1 gene, encoding a potential prenyltransferase, are causal for Schnyder crystalline corneal dystrophy. PLoS One 2: e685, 2007. 